\title{
A Wireless Implantable Micropump for Chronic Drug Infusion Against Cancer
}

\author{
Angelica Cobo ${ }^{1}$, Roya Sheybani ${ }^{1}$, Heidi Tu ${ }^{1}$, Ellis Meng ${ }^{1,2,3}$
}

${ }^{I}$ Department of Biomedical Engineering, Viterbi School of Engineering, University of Southern California, 1042 Downey Way, DRB-140, Los Angeles, CA 90089-1111, USA

${ }^{2}$ Department of Electrical Engineering, Viterbi School of Engineering, University of Southern California, 3651 Watt Way, VHE-602, Los Angeles, CA 90089-0241, USA

${ }^{3}$ Corresponding Author: Ellis Meng

Phone: (213) 740-6952

Fax: (213) 821-3897

E-mail: ellis.meng@usc.edu

\begin{abstract}
We present an implantable micropump with a miniature form factor and completely wireless operation that enables chronic drug administration intended for evaluation and development of cancer therapies in freely moving small research animals such as rodents. The low power electrolysis actuator avoids the need for heavy implantable batteries. The infusion system features a class $\mathrm{E}$ inductive powering system that provides on-demand activation of the pump as well as remote adjustment of the delivery regimen without animal handling. Micropump performance was demonstrated using a model anti-cancer application in which daily doses of $30 \mu \mathrm{L}$ were supplied for several weeks with less than $6 \%$ variation in flow rate within a single pump and less than $8 \%$ variation across different pumps. Pumping under different back pressure, viscosity, and temperature conditions were investigated; parameters were chosen so as to mimic in vivo conditions. In benchtop tests under simulated in vivo conditions, micropumps provided consistent and reliable performance over a period of 30 days with less than $4 \%$ flow rate variation. The demonstrated prototype has potential to provide a practical solution for remote chronic administration of drugs to ambulatory small animals for research as well as drug discovery and development applications.
\end{abstract}

Keywords: Drug delivery; wireless powering; implantable micropumps; MEMS electrochemical bellows actuator; check valve; cancer

1

${ }^{1}$ Abbreviations: siRNA, Small interfering ribonucleic acid; Pt, Platinum; PBS, Phosphate buffered saline; PDMS, Polydimethylsiloxane; PEG, Polyethylene glycol; USP, United States Pharmacopeia; SL, Stereolithography; EI, Electrochemical impedance 


\section{Introduction}

Each year, approximately 8 million people around the world die from cancer Research suggests that one-third of these deaths can be prevented with appropriate technologies and services (Centers for Disease Control and Prevention 2015). Cancer treatment typically involves surgery to remove cancerous tissues, followed by long-term chemotherapy or radiation therapy, among other types of therapies (National Cancer Institute 2015). Chemotherapy and radiotherapy are used to initiate intrinsic apoptotic (cell death) pathways, but these type of treatments, when administered systemically, are non-specific and affect non-cancerous tissues as well as leading to severe side effects. Delivery to a specific tissue facilitates the goal of achieving therapeutic drug levels while reducing harmful side effects, the amount of drug needed, and the frequency of dosing events (Langer 1998). Additionally, studies have shown that periodic biological fluctuations such as circadian rhythms may affect drug kinetics and dynamics (Smolensky and Peppas 2007). Administration of chemotherapy at a specific time in a tumor cell cycle can increase treatment effectiveness while decreasing toxicity to healthy tissue (Youan 2004). A study of more than 30 anticancer drugs showed that the toxicity and efficacy of these drugs varied by more than $50 \%$ depending on the time of dosing (Lévi, Focan et al. 2007). Patient tolerance to medication and clinical outcome are also greatly affected by the dosing time (Lévi, Focan et al. 2007). Greater control over delivery rates allows tailored therapies and drug regimen changes during the course of therapy. Consequently, there is a great need for therapies developed and combined with delivery methods to allow for long-term, localized, and patient tailored anti-cancer therapies.

In drug development for disease management, the scientific community relies heavily on animal studies to provide basis for human clinical trials (Reagan-Shaw, Nihal et al. 2008). The mouse genome has been completely sequenced allowing accurate comparisons to other mammal genomes such as humans and its physiologic, metabolic and pathologic processes have been well documented (Linder and Davisson 2012). As such, human drug therapies are initially evaluated in small animal models; therefore, drug administration technologies for small laboratory animals are critically important for drug discovery and development. The most commonly used drug 
administration routes for laboratory animals are oral, topical, or intravenous which require high drug dose concentration, leading to severe side effects, and frequent animal handling. In chronic studies, frequent animal handling or restraint induces stress, which can confound study results (John Urquhart 1984).

Current technologies for chronic dosing in laboratory animals include tethered infusion systems and vascular access ports. In tethered systems, the animal is connected to a precision infusion pump with a tether as shown in Fig. 1a (Turner, Brabb et al. 2011). The use of the external pump provides accurate dosing, but is impractical for chronic studies since restriction of movement by the tether induces stress. Vascular access ports are chronically implanted subcutaneously minimizing animal movement restrictions (Turner, Pekow et al. 2011). However, vascular ports require regular cleaning, maintenance, and appropriate protective measures to prevent infection or damage by the animal.

Implantable infusion pumps further minimize animal handling and permit natural movement after the initial healing period following implantation. The Med-e-Cell Infu-Disk is non-refillable, has a preset constant flow rate, and a maximum lifetime of 1 week (Med-E-Cell). This pump is only suitable for drug delivery in animal models larger than rats due to its large size. The Primetech iPrecio and the Alzet pumps are the only commercial pumps scaled for mice. The iPrecio peristaltic pump allows programmable infusion profiles, but is battery powered, only provides up to 52 days of operation, cannot be powered off, and is single use (iPrecio). The Alzet osmotic pump provides a preset continuous flow rate, a drug payload of up to 6 weeks, and is non-refillable (Alzet). These technical limitations of current technology prohibit flexibility in designing administration regimens for drug discovery and development research. While commercially available pumps are not suitable for chronic drug infusion in small animals, recent advances in microelectromechanical systems (MEMS) technology have led to the miniaturization and improved efficacy of implantable drug delivery systems suitable for small laboratory animals (Meng and Hoang 2012). Several ongoing efforts are being carried out in research laboratories to develop remotely activated drug delivery microsystems. Detailed descriptions of these efforts are available in (Sheybani, Schober et al. 2013, Cobo, Sheybani et al. 2015). 
The micropump presented in this work was actuated using electrolysis which offers low power consumption, large driving force, low heat generation, on-demand activation, and post-implantation flow rate control through the applied current (Sheybani, Gensler et al. 2012). Wired implantable MEMS micropumps scaled for use in small laboratory animals (e.g., mice) were previously demonstrated (Gensler, Sheybani et al. 2010, Gensler, Sheybani et al. 2012, Sheybani, Gensler et al. 2012). Preliminary in vivo studies with this wired system demonstrated the potential of using micropumps to combat radiation resistance tumor in mice by improving the exposure of tumors to siRNA through sustained localized delivery compared to needle injections (Gensler, Sheybani et al. 2010, Gensler, Sheybani et al. 2012, Masood, Roy et al. 2012). However, the use of transcutaneous wires for powering the pump required animals to be restrained during dosing, prohibited chronic studies, and placed restrictions on experimental throughput. Also, the lack of reliable, small form factor check valves resulted in a slow diffusion of drug from the reservoir into the animal and thereby limited the accuracy of delivery. The absence of a reliable check valve could also lead to fluid to flow back through the delivery catheter to the reservoir as a result of the reverse pressure gradient created during recombination of electrolysis gases.

Here, we present a redesigned implantable micropump that incorporates wireless power telemetry and an integrated miniature check valve, suitable for chronic drug delivery in untethered, freely moving small laboratory animals such as mice (Fig. 1b, Table 1). This wireless drug administration method can eliminate the induced stress from animal handling or tethered and wired drug administration systems that could potentially confound interpretation of data (John Urquhart 1984). Wireless operation was facilitated by improving electrolysis actuation efficiency which further reduced actuator power consumption ( $1 \mathrm{~mW}$ from $3 \mathrm{~mW}$ ). A miniature check valve with small form factor and improved performance enabled appropriate flow regulation during pumping and resting states. The check valve contributes to accurate dosing and prevents drug contamination with biological fluids during the recombination reaction between electrolysis actuation cycles (Sheybani, Gensler et al. 2012). Acute characterization of pump performance was previously presented (Cobo, Tu et al. 2014). For this work, the pump performance characterization was extended to 
simulate in vivo chronic conditions, including those relevant to future studies to deliver agents to combat radiation resistance in a mouse xenograft cancer model. A redesigned drug reservoir achieved a more compact footprint by reducing dead volume (inaccessible drug) by nearly $91 \%$ and check valve dimensions, which were essential to accommodate the addition of wireless circuitry. Integrated refill ports allow long term use of the micropump for chronic studies.

(a)

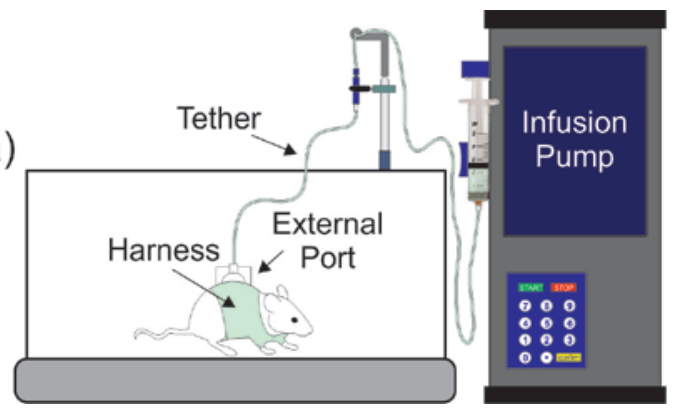

(b)

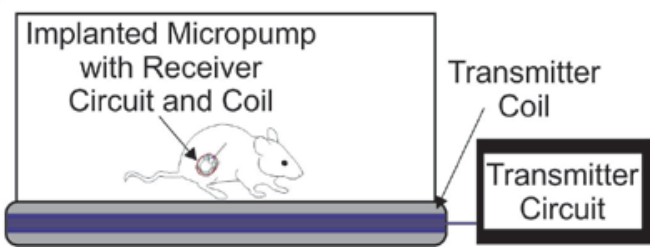

Fig. 1. Schematic diagram of in vivo testing setup for small animal research with (a) commercial tethered infusion system, and (b) wireless implantable drug delivery pump

Table 1

Primary differences between the previous generation refillable implantable micropump system (RIMS) and our current implantable micropump for drug delivery in small animals

\section{RIMS Pump (Gensler, Sheybani et al. 2012) \\ Current Pump}

Operation

One way valve specifications

Height

Footprint

Dead volume

Power consumption
Wired, External constant current power supply

Bridge-type membrane valve

0.515 x $1.23 \mathrm{~mm}, \mathrm{~L}$ x OD

$1.31 \mathrm{kPa}$ cracking pressure

$10 \mathrm{~mm}$

$21 \mathrm{~mm} \mathrm{OD}$

$1087 \mu \mathrm{L}$

$3 \mathrm{~mW}$
Wireless power telemetry

Duckbill valve

$3 \times 2 \mathrm{~mm}, \mathrm{~L} \times \mathrm{OD}$

$0.69 \mathrm{kPa}$ cracking pressure

\begin{tabular}{|cc|}
\hline $10 \mathrm{~mm}$ & $8.1 \mathrm{~mm}$ \\
\hline $21 \mathrm{~mm} \mathrm{OD}$ & $20 \times 15 \mathrm{~mm}, \mathrm{~L} \mathrm{x} \mathrm{W}$ \\
\hline $1087 \mu \mathrm{L}$ & $96 \mu \mathrm{L}$ \\
\hline $3 \mathrm{~mW}$ & $1 \mathrm{~mW}$ \\
\hline
\end{tabular}

\section{Design}

Our micropump system utilizes a low power electrolysis actuation mechanism. The electrochemical actuator consists of an electrolyte (water) encased by a Parylene bellows and a pair of interdigitated platinum $(\mathrm{Pt})$ electrodes supported on a rigid glass substrate. An applied electrical current to the electrodes dissociates liquid water into 
oxygen and hydrogen gas and induces an increase in pressure. This deflects the bellows, activates a one-way check valve, and displaces the surrounding fluid out of the rigid reservoir through the outlet catheter to the delivery site. The gases recombine to water once the current is turned off, allowing the bellows to return to the original rest position.

The interdigitated electrode design reduces the solution resistive path resulting in improved electrolysis reaction ( $\mathrm{Li}$, Sheybani et al. 2010). Pt was selected as the electrode material due to its biocompatibility, good corrosion and oxidation resistance, and ability to catalyze recombination (Ioroi, Yasuda et al. 2002, Grigoriev, Millet et al. 2010). Electrolysis efficiency was further improved by coating the electrodes with Nafion $\AA$, a solid polymer electrolyte that provides higher solubility of oxygen and hydrogen gases than water (Sheybani, Gensler et al. 2012). Nafion ${ }^{\circledR}-$ coated electrodes also provide faster and more repeatable recombination, decrease electrode delamination or damage at high current operation, and increase flow rate (Sheybani and Meng 2012).

Bellows possess the ability to achieve large deflection with low applied pressure compared to corrugated or flat diagrams (Li, Sheybani et al. 2010). Parylene C was selected as the bellows material due to its low Young's modulus, biocompatibility, inertness and low permeability ( $\mathrm{Li}$, Sheybani et al. 2010, Ghodssi and Lin 2011). The bellows consisted of two convolutions each having a $6 \mathrm{~mm}$ inner diameter, $9.5 \mathrm{~mm}$ outer diameter, $0.4 \mathrm{~mm}$ height and $13.5 \mu \mathrm{m}$ thickness. This bellows can safely displace $185 \mu \mathrm{L}$ of fluid contained within the adjacent drug reservoir without experiencing plastic deformation (Gensler and Meng 2012).

The micropump design achieves compact footprint by reducing dead volume between the reservoir wall and bellows. The reservoir inner diameter was reduced by $46 \%$ resulting in a substantial reduction in dead volume from approximately 1100 to $100 \mu \mathrm{L}$ compared to previous micropump prototypes (Gensler, Sheybani et al. 2013); this new volume combined with the capability for refill is adequate for most small animal drug delivery applications. The reduction in reservoir footprint allowed incorporation of the wireless components while maintaining a total device mass of 
approximately $10 \%$ of the weight of the animal (typically adult mice are $30 \mathrm{~g}$ ) to allow free, unhindered movement (The Johns Hopkins University 2015).

The pump is normally off until wirelessly activated by a class E inductive powering system (Fig. 2). Inductive transmission is most suitable for transcutaneous powering compared to other forms of wireless powering (radiation, conductive, and capacitive) (Lenaerts and Puers 2009). For this system the power transmission frequency was chosen to minimize heat generation (Givrad 2007). It relies on magnetic field coupling and transmission frequency can be chosen to minimize power dissipation. The class $\mathrm{E}$ inductive powering transmitter is placed underneath the rodent cage. A current regulator on the subcutaneously implanted receiver controls the application of current to the electrolysis actuator; here, a single constant current magnitude was supplied corresponding to a single flow rate. The volume delivered is controlled by the duration of pump activation. Heat generation from the wireless system is negligible in part due to chosen operation frequency (Sheybani, Schober et al. 2013). High efficiency off the shelf components were chosen to minimize power dissipation (van Schuylenbergh and Puers 2009).

Transmitter

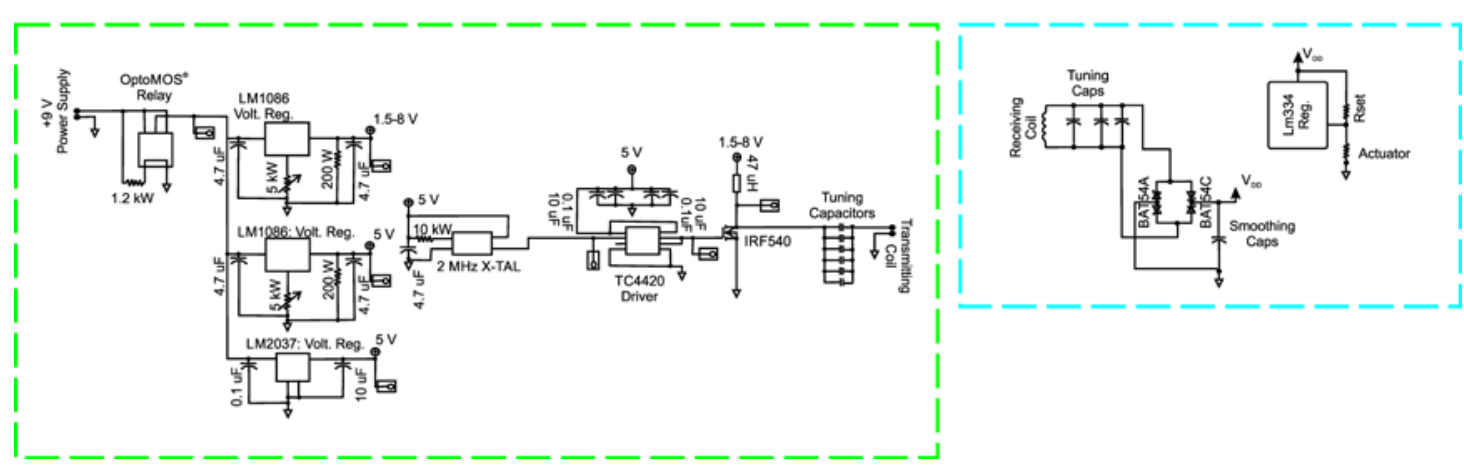

Fig. 2. Circuit schematic for the class E wireless inductive powering system. Link efficiency for concentric transmitting and receiving coils with zero angular misalignment was calculated to be approximately 0.82 .

\section{Fabrication}

\subsection{Electrolysis Actuator}

The actuator's interdigitated electrodes $(100 \mu \mathrm{m}$ wide elements separated by 100 $\mu \mathrm{m}$ gaps, $8 \mathrm{~mm}$ diameter footprint, $300 \AA / 2000 \AA \mathrm{Ti} / \mathrm{Pt}$ ) were fabricated on a 
Borofloat 33 glass substrate (University Wafer, Boston, MA) by liftoff following ebeam deposition of the thin film metal using a previous method described in (Gensler, Sheybani et al. 2012, Sheybani and Meng 2012). The electrodes were potentiostatically cleaned at $\pm 0.5 \mathrm{~V}$ (Gamry Reference 600 Potentiostat, Warminster, $\mathrm{PA}$ ) in $1 \mathrm{X}$ phosphate buffered saline (PBS). The electrodes were then dip coated in Nafion ${ }^{\circledR}$ (Dupont DE521 Solution, Ion Power, INC, New Castle, DE). Kynar ${ }^{\mathrm{TM}}$ nickel plated copper wires (30 AWG, Jameco Electronics, Belmont, CA) were soldered to the electrode contact pads. The joint was reinforced and insulated with nonconductive marine epoxy (Loctite, Westlake, OH) (Sheybani and Meng 2012).

Parylene bellows were fabricated using a lost-wax two-part molding process previously described in (Gensler and Meng 2012). Polydimethylsiloxane (PDMS, Sylgard 184; Dow Corning Corp., Midland, MI) sheets (0.4 mm thick) were perforated with $6 \mathrm{~mm}$ and $9.5 \mathrm{~mm}$ holes to mold the inner and outer diameters of the bellows, respectively. The sheets were visually aligned and stacked together on glass slides to form mold modules. These modules were filled with molten $\left(50^{\circ} \mathrm{C}\right)$ polyethylene glycol (PEG; 1,000 Mn, Sigma Alrich, St. Louis, MO). Once the PEG solidified, PDMS sheets were peeled. The modules were stacked and fused together by moistening connecting interfaces with water. Completed PEG molds were coated with $13.5 \mu \mathrm{m}$ United States Pharmacopeia (USP) class VI Parylene C (Specialty Coating Systems, Indianapolis, IN), followed by soaking in deionized water at room temperature to remove sacrificial PEG.

The Parylene $\mathrm{C}$ bellows were filled with double deionized water and attached to the electrode using double-sided pressure sensitive adhesive film (Tape 415, 3M, St. Paul, MN). The joint was then reinforced with marine epoxy.

\subsection{Check Valve}

A one-way duckbill valve made of medical grade silicone $(2.0 \mathrm{~mm}$ duckbill; Minivalve, Cleveland, $\mathrm{OH}$ ) with a measured cracking pressure of less than $0.69 \mathrm{kPa}$ $(5.17 \mathrm{mmHg})$ was selected. Valves were prescreened for proper closure and then incorporated into the pump by attaching the base of the valves to the reservoir 
housing output path using biocompatible epoxy (EPO-TEK ${ }^{\circledR} 730$ unfilled, Epoxy Technologies, Billerica, MA).

\subsection{Wireless Powering System}

A $2 \mathrm{MHz}$ clock oscillator (ECS -2100, ECS international, Olathe, KS) was used to create the power signal from a $9 \mathrm{~V}$ power supply $(0.3 \mathrm{~W})$. The generated signal was then amplified in two stages before being applied to a tuned transmitting coil ( 8 turns of 20 AWG single strand wire, size: $310 \mathrm{~mm}$ x $140 \mathrm{~mm}$ ). Litz wire (6 turns, 50/54 SPN/SN Litz Wire, Wiretron, Volcano, CA) was used for the receiving coil (Ø 17 $\mathrm{mm})$. The received alternating signal was fully rectified using two Schottky diodes (BAT54A and BAT54C, Fairchild Semiconductor, San Jose, CA). A resistor was used to set the output current on a 3-terminal adjustable current source (LM334, Texas Instruments,

Dallas, TX) to $0.33 \mathrm{~mA}$ for driving the pump.

\subsection{System Packaging and Assembly}

The reservoir domes and bases were produced using stereolithography (SL) in USP class VI DSM Somos ${ }^{\circledR}$ WaterShed XC 11122 material (FineLine Prototyping, Inc., Raleigh, NC). This material is optically clear, durable, water resistant, and rigid (Somos 2008, DSM Somos Materials Group 2012).

Medical grade silicone rubber septa (10:1 base-to-curing agent ratio USP class VI MDX-4 4210; Factor II, Lakeside, AZ) were fabricated based on results of a leak puncture test allowing for at least one dozen punctures with a maximum applied pressure within the dome of $775 \mathrm{mmHg}(15 \mathrm{psi})$. The septa were then inserted into the refill ports of the SL dome and the perimeters reinforced with marine epoxy. This assembly was encapsulated with $5 \mu \mathrm{m}$ Parylene $\mathrm{C}$ to further improve biocompatibility and barrier properties. Then the electrolysis actuator was situated in the reservoir assembly and secured with biocompatible epoxy.

The wireless receiver was mounted on the micropump body and encapsulated with $5 \mu \mathrm{m}$ Parylene $\mathrm{C}$, followed by a thin coating of medical grade silicone to provide a 
softer interface with tissue. The total mass of a filled prototype pump was $3.8 \mathrm{~g}$ (Fig. 3).

(a)

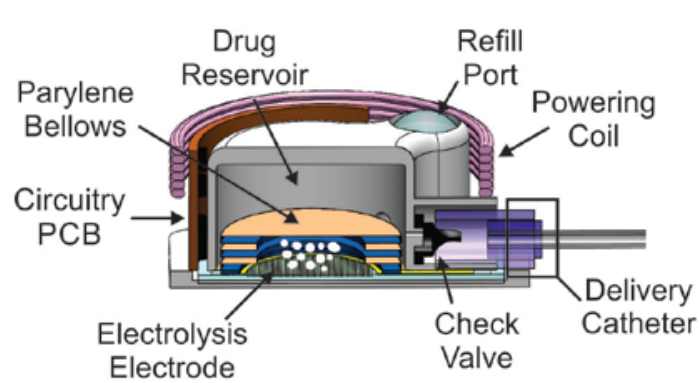

(b)

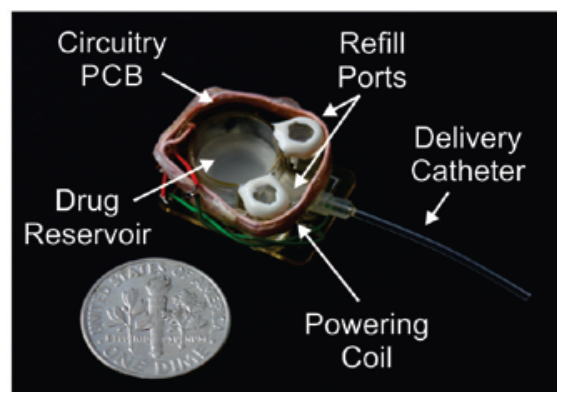

Fig. 3. Schematic diagram of (a) drug delivery system, and (b) fully assembled wireless micropump

\section{Experimental Methods}

Our specific application of interest is a micropump for a siRNA-based therapy against radiation resistant cancer in which an electrostatically-coupled siRNA-gold nanorod drug is directly delivered to xenograft tumors in nude mice. This anti-cancer application requires low flow rate $(\sim \mu \mathrm{L} / \mathrm{min}), 30 \mu \mathrm{L}$ daily dose volume, and infusion duration of at least two weeks with one refill per week. The micropump's ability to administer the desired regimen under wireless operation was investigated on benchtop. In addition, the repeatability of performance across different wireless receivers and impact on performance of changes in coil orientation were examined. Power transfer can be affected by coil orientation changes. The wireless micropump is intended to be implanted in a freely moving animal. Therefore, it is important to investigate the effects of varying the axial separation distance and angular misalignment between the transmitting and receiving coils that can result over the course of drug administration in an animal study. Previously, we presented preliminary results of successful micropump performance in simulated in vivo conditions including delivery against a physiologically relevant back pressure and delivering solutions of differing viscosities (Cobo, Tu et al. 2014). Here, we extend this simulated in vivo studies to analyze micropump performance when delivering at body temperature and long term pump functionality when subjected to a simulated in vivo environment (saline soak at body temperature). 


\subsection{Wireless Testing}

Flow rate measurements were taken on a single micropump with five different wireless receivers. Flow rate was calculated by measuring fluid front displacement in a $100 \mu \mathrm{L}$ calibrated micropipette (VWR International, Radnor, PA) connected to the catheter outlet as shown in Fig. 4. The axial separation distance (parallel coils, $2-4$ $\mathrm{cm}, 0.5 \mathrm{~cm}$ steps) and angular misalignment $\left(0,30,45\right.$, and $\left.60^{\circ}\right)$ between concentric transmitter and receiver coils ( $2 \mathrm{~cm}$ distance between coils) was varied to study the effects on pump flow rate performance.

\subsection{Daily Dosing}

Successful dose delivery of six stationary $\left(2 \mathrm{~cm}\right.$ distance and $0^{\circ}$ angular misalignment between concentric coils) micropumps over a period of one week was obtained. Here, two of the previous micropumps were operated daily for an additional week to simulate the two week dosing regimen planned for the anti-cancer application. Daily dosing regimen over a period of three weeks was studied in one of said micropumps. A constant current of $0.33 \mathrm{~mA}$ was supplied to the actuator wirelessly for a duration that allowed $30 \mu \mathrm{L}$ of double deionized water to be delivered daily. The pumps were only refilled once per week and the duration of applied current was increased with each successive dosing event. The micropump catheter was connected to a calibrated micropipette for flow rate measurements as previously described. The fluid front position was recorded several times during each delivery at discrete time points. Reverse leakage between dosing events, if any, was recorded. To avoid confounding results from evaporation, the fluid front being tracked was followed by a small air pocket $(10 \mathrm{~mm})$, followed by a column of water resulting in negligible evaporation between dosing events.

\subsection{Room Temperature Versus Body Temperature}

In implantable drug delivery devices, the device will be operated at body temperature. Three fully assembled and encapsulated micropumps were filled with double deionized water, flush of any air bubbles and soaked in a room $\left(23^{\circ} \mathrm{C}\right)$ or body temperature $\left(37^{\circ} \mathrm{C}\right)$ deionized water bath for 30 minutes. Flow rate test was 
performed while the pump was submerged in a temperature-controlled water bath. The flow rate was determined as previously described during four cycles each lasting 5 minutes after valve opening.

\subsection{Physiological Enviroment Simulation}

System performance in simulated in vivo conditions was investigated to verify that Parylene $\mathrm{C}$ and silicone rubber encapsulation layers provide adequate protection for the electronics for the duration of the anticipated in vivo study. Three fully assembled and encapsulated micropumps were soaked in $1 \mathrm{X}$ PBS at body temperature $\left(37^{\circ} \mathrm{C}\right)$. Periodically, the micropumps were removed from the bath, refilled with double deionized water, and subjected to flow rate testing. Valve opening times were recorded and flow rate measurements were taken for 5 minutes after valve opening.

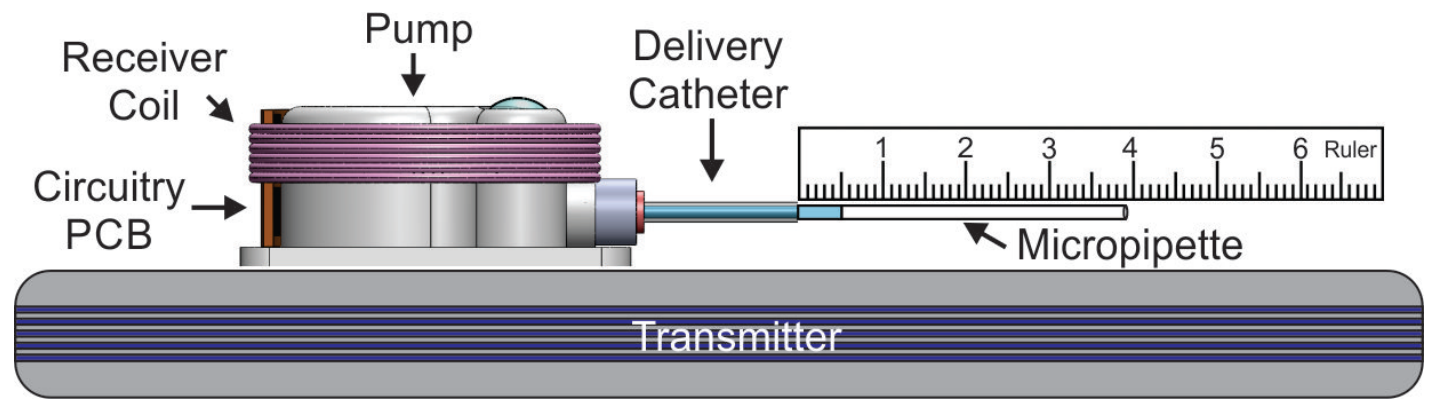

Fig. 4. Experimental setup for flow rate characterization of wireless micropumps

\section{Experimental Results}

\subsection{Wireless Testing}

Benchtop testing of a single micropump with five different receivers resulted on an average flow rate of $2.66 \pm 0.16 \mu \mathrm{L} / \mathrm{min}$ (mean $\pm \mathrm{SE}, n=5$ ), a $6 \%$ variation between receivers. The results from changing the axial separation distance between coils and angular misalignment between transmitter and receiver showed a drop in flow rate by $64.1 \%$ and $42.86 \%$ for a $3.5 \mathrm{~cm}$ separation distance between coils and for $45^{\circ}$ angular misalignment between transmitter and receiver, respectively as shown on Fig. 5. 
(a)

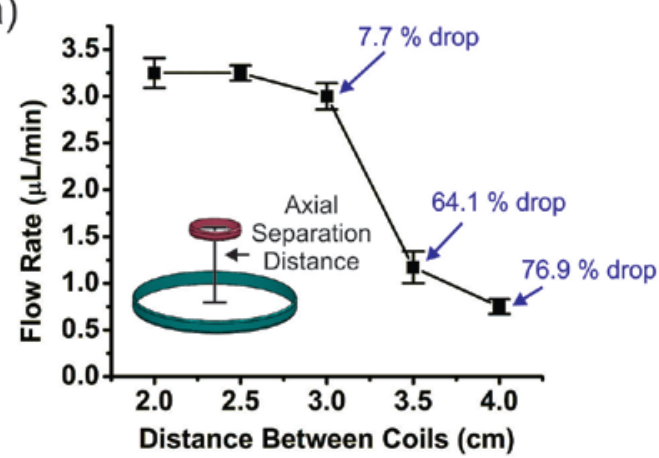

(b)

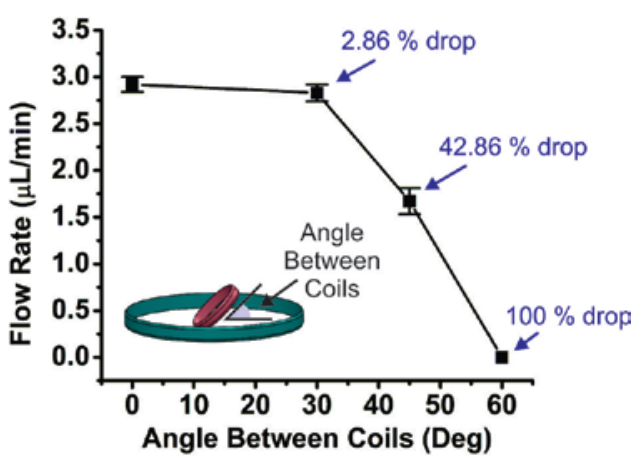

Fig. 5. The effects of (a) axial separation distance between concentric transmitter and receiver coils, and (b) angular misalignment between concentric coils on pump performance (at $0.33 \mathrm{~mA}$ constant current)

\subsection{Daily Dosing}

Two wirelessly operated micropumps consistently delivered $30 \mu \mathrm{L}$ doses daily over the course of two weeks as required by the anti-cancer application. These micropumps showed similar flow rate performance during the two week testing with less than $8 \%$ variation. Fig. 6a shows representative results from the daily dosing regimen of one micropump for one week. The data is presented as accumulated volume to study the effects of increasing pressure build up within the reservoir during recombination periods. Similar data was obtained for all pumps tested. The shown micropump delivered $30 \mu \mathrm{L}$ doses daily for three consecutive weeks. Mean flow rates during the first two weeks are not significantly different as confirmed by statistical analysis (ANOVA, $\mathrm{p}>0.05$ ) (Fig. 6b). Valve performance varied across the micropumps, but it consistently limited back flow to a maximum of $10 \mu \mathrm{L}$ when the pump was turned off. The slight volume drop seen before each dosing event correlates to the back flow allowed by the valve. Some valves showed improved sealing performance in each consecutive week of testing. 
(a)

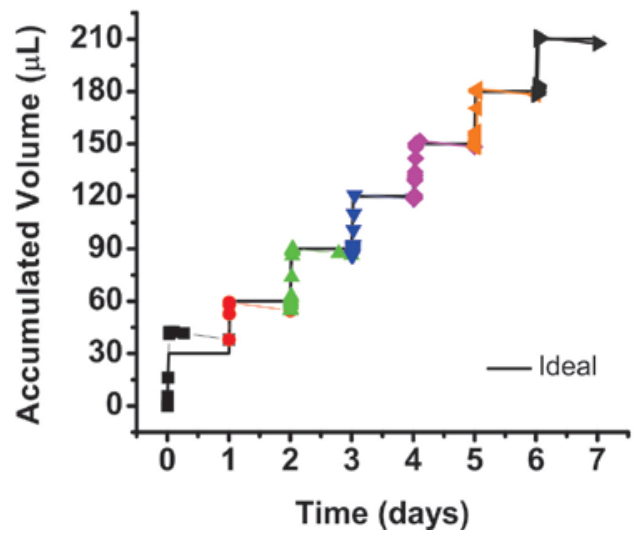

(b)

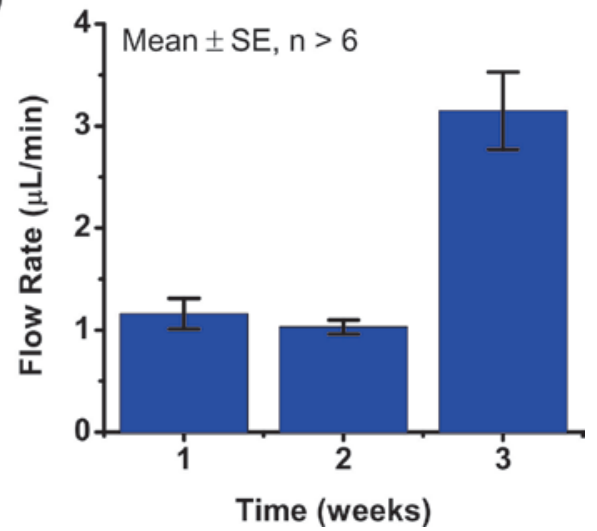

Fig. 6. Representative data from one stationary micropump wirelessly operated. (a) Bolus delivery from a micropump operated once a day during week two (at $0.33 \mathrm{~mA}$ constant current) and (b) flow rates for the three weeks dosing

\subsection{Room Temperature Versus Body Temperature}

Fig. 7 shows representative results of the flow rate performance at room temperature $\left(23^{\circ} \mathrm{C}\right)$ and body temperature $\left(37^{\circ} \mathrm{C}\right)$ for one micropump (mean $\pm \mathrm{SE}, n$ $=4)$. As expected, an increase in flow rate was apparent at body temperature. For two micropumps, changes in temperature resulted in significant flow rate differences as confirmed by performing a one way analysis of variance (ANOVA, $\mathrm{p}<0.05$ ).

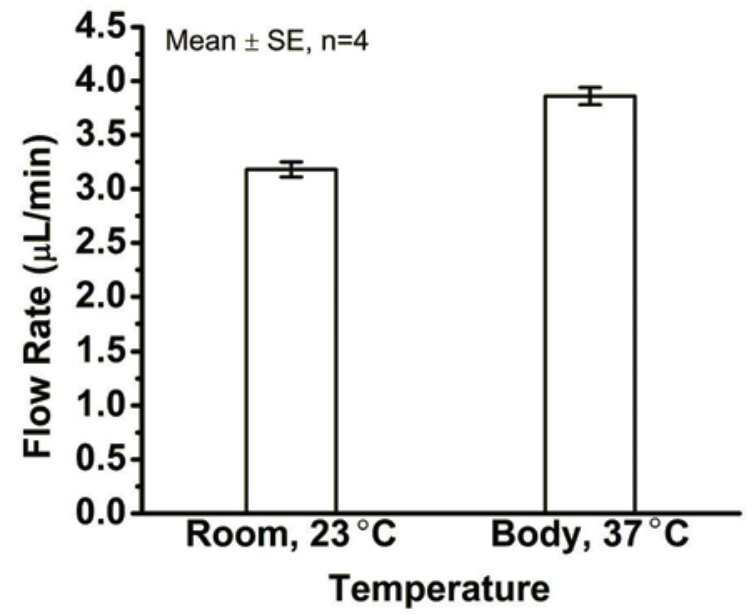

Fig. 7. Representative data from one wireless micropump flow rate performance when increasing the environmental temperature from room $\left(23{ }^{\circ} \mathrm{C}\right.$ ) to body temperature $\left(37^{\circ} \mathrm{C}\right.$ ) (at $0.33 \mathrm{~mA}$ constant current)

\subsection{Physiological Enviroment Simulation}

A 30 day trial with three fully encapsulated and packaged pumps immersed in a $1 \mathrm{X}$ PBS bath at body temperature $\left(37^{\circ} \mathrm{C}\right)$, showed continued accurate and reliable flow 
rate performance with a standard error of less than $4 \%$ for each pump. Fig. 8 shows representative results of the flow rate performance for one micropump (mean $\pm \mathrm{SE}, n$ =4). This micropump was previously used for the three week daily dosing study, but has a new receiver. Mean flow rates between weeks for each pump are not significantly different as confirmed by statistical analysis (ANOVA, $\mathrm{p}>0.05$ ).

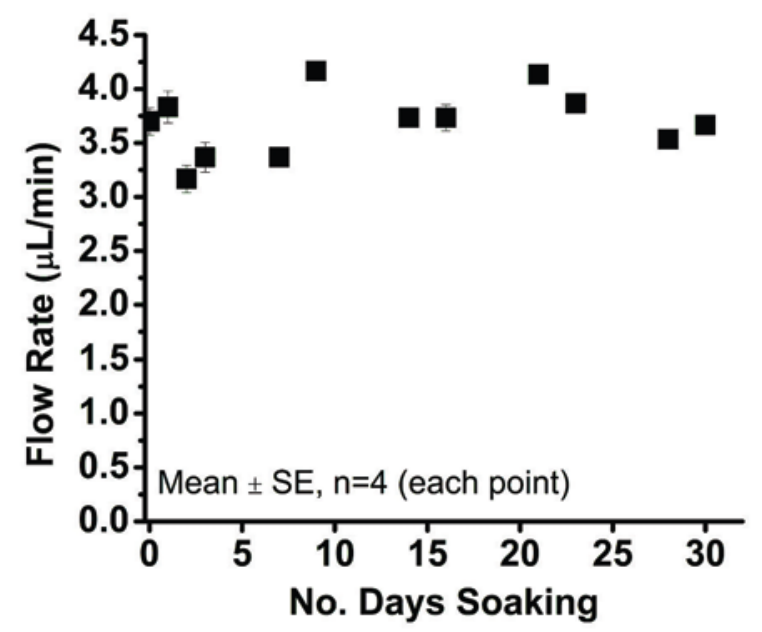

Fig. 8. Representative flow rate performance results for a micropump under simulated in vivo conditions for 30 days (at $0.33 \mathrm{~mA}$ constant current)

\section{Discussion}

The pump components were redesigned to optimize pump performance and ease of use. The new reservoir dome features two refill ports as oppose to one to facilitate filling and flushing of the reservoir. The refill ports are robust enough to prevent leaks after high number of punctures under high reservoir pressure. The new redesigned rigid reservoir significantly decreased device footprint by decreasing dead volume by $91 \%$. The total filled encapsulated device mass including wireless power components was $3.8 \mathrm{~g}$, slightly exceeding the mass requirements for a mouse experiment ( $3 \mathrm{~g}$ or approximately $10 \%$ of an adult mouse weight of $30 \mathrm{~g}$ ). The encapsulation was identified as the heaviest component. Therefore, the pump mass can be reduced by eliminating the encapsulation material and instead using a low density polymer outer casing to enclose the current system.

Fully integrated wireless stationary micropumps successfully delivered $30 \mu \mathrm{L}$ daily boluses at low flow rates $(\sim \mu \mathrm{L} / \mathrm{min})$ for several weeks, a regimen relevant for the administration of siRNA therapy to a radiation resistance cancer. The 2.8 fold 
increase in flow rate observed during the third week of the daily dosing study was attributed to a faulty receiver. Due to this systematic error, flow rate variation was calculated after receiver was replaced (4\%). Since we previously demonstrated consistent actuator performance (Sheybani, Gensler et al. 2012), slight variations in mean flow rate were attributed to variations due to manual assembly of the packaging and receiver coils and inconsistent performance in the commercially produced valves. Also, valves permitted up to $33 \%$ of dose volume in back flow when the pump was turned off. Some delay in forward flow during current application was present in all micropumps tested and is due to the low current application (Neagu 1998), and valve cracking pressure. This delay was further increased in daily dosing regimens where the reservoir was not refilled and higher pressure build up was required in order to deliver the same dose volume (Sheybani, Gensler et al. 2012). As discussed in (Gensler 2013), the forward flow delay prior to refill follows a predictable pattern and can be accounted for by increasing the current application time, thus our flow rate variation of $4 \%$ does not include the forward flow delay. The forward flow delay in our previous prototypes was minimized by replacing the compliant reservoir material with a rigid material and utilizing higher efficiency electrolysis electrodes (Gensler, Sheybani et al. 2012, Sheybani and Meng 2012). The dosing regimen can be altered depending on the intended application. A diverse assortment of liquid drug formulations can be delivered within a wide dynamic range of dose volumes $(\mathrm{nL}-$ $\mu \mathrm{L})$ and flow rates $(0.33-141.9 \mu \mathrm{l} / \mathrm{min})$ (Sheybani, Gensler et al. 2012). The bellows can safely deliver $180 \mu$ l. Once this volume is reached the actuator should be allowed to fully recombine prior to the next dosing event. Full recombination is achieved within several hours (Sheybani and Meng 2015). While the pump can store medication for long term, it is important for the drug to be stable when stored at body temperature between refill events.

Previous prototypes of this pump included commercial valves with high sealing pressures (Gensler, Sheybani et al. 2012) that showed significant reverse flow during recombination. For in vivo studies reverse flow needs to be avoided in order to obtain safe and accurate dosing. The check valve incorporated into this systems was the only small commercial valve with low sealing pressure and cracking pressure. Unfortunately, the valve manufacturing process leads to variability and inconsistency 
in valve performance. Thus, valves were screened before incorporation into the pump and only $50 \%$ of the valves passed screening (reverse leakage $<20 \%$ of dose volume). Variable and inconsistent performances of the valve resulted in additional variability among pump performance. Therefore, high quality valves with repeatable performance are now being developed.

A class D wireless power system was developed to power an electrochemical actuator in (Sheybani, Gensler et al. 2012). While it was simple in design and provided large currents, this class D system had several drawbacks including difficulty in fine tuning the amplifier and inability to generate the required power without overheating the transmitter circuit components. A class E system combines the advantages of the class D system with its series-tuned output and parallel-tuned amplifier tanks. Class E systems can cope with the rigorous demands of weakly coupled links, produce smooth signals, be insensitive to low switching time, and operate over several tens of MHz. This pump was powered with a class E powering system, which allows higher efficacy when a considerable amount of power is to be transferred (Puers 2008). Pump flow rate is linearly dependent on applied current to the actuator (Sheybani and Meng 2012). A current regulator was chosen as part of the implanted receiver circuit to ensure the current applied to the actuator to be relatively constant (within certain limits of distance and angular misalignment between the transmitting and receiving coils) regardless of slight changes in the power. Current regulators are inherently lossy components, as such, power efficiency is partially sacrificed to ensure proper pump operation. Flow rate performance for an individual pump varied when it was powered with different receivers. Variations in flow rate remained within less than $6 \%$. This slight variation in performance can be attributed to the manual assembly of receiver coils, but can be alleviated by calibrating each pump prior to in vivo studies. As expected, a decrease in power transferred due to increased axial separation distance and angular misalignment between concentric transmitter and receiver coils resulted in decreased in flow rate. Pump flow rate stability can be further improved by increasing the transmitted power (Lenaerts and Puers 2009). The addition of multiple coils at either the transmitter or receiver side can improve power transfer to mitigate angle misalignment. Another potential solution is to move the external receiving coil along with the moving animal by 
tracking the movement of the internal transmitting coil with a similar setup to the one proposed by Kilinc, et. al. (Kilinc, Kapucu et al. 2014).

The effects of environmental factors on pump performance were investigated. Changing the environmental temperature from room temperature $\left(23{ }^{\circ} \mathrm{C}\right)$ to body temperature $\left(37^{\circ} \mathrm{C}\right)$ for our wireless micropumps resulted in increased flow rate. Improved electrolysis efficiency can be attributed to the increased permeability of Nafion ${ }^{\circledR}$ to hydrogen and oxygen when the ambient temperature is increased (Broka and Ekdunge 1997). This is in agreement with (Sheybani, Gensler et al. 2012), which found slight increase in flow rate at body temperature for electrochemical actuators. Physiological environmental simulation test showed that all three pumps were functioning consistently and as expected after 30 days of soaking in $1 \mathrm{X}$ PBS at body temperature $\left(37^{\circ} \mathrm{C}\right)$ exceeding the intended in vivo study duration by $100 \%$. This verifies that the Parylene $\mathrm{C}$ and silicone rubber encapsulation layers provide adequate protection for the electronics for the duration of the anticipated in vivo study. This packaging scheme may also be suitable for longer term in vivo studies beyond 30 days.

\section{Conclusion}

We demonstrated the characterization of a fully implantable wireless infusion micropump for use in chronic drug delivery therapies in small research animals. Wireless control of operation permits a user to select the desired delivery regimen in a freely moving animal. Wireless powering of the actuator with a class $E$ inductive powering system allowed repeatable delivery with less than $6 \%$ variation in flow rate. Pump performance was affected by increased axial separation distance and angular misalignment between concentric transmitter and receiver coils. If necessary, this can be alleviated by increasing the power transmitted and inclusion of a second receiver coil (Lenaerts and Puers 2009). Wireless powering with an implantable device requires inductive coupling link through the skin and biological tissue. As such, it is essential to characterize device performance in vivo. Future work will include characterization of system operation in simulated tissue material to mimic device operation in animals. 
Stationary micropumps successfully delivered a single bolus daily for several weeks and achieved flow rates on the order of $\mu \mathrm{L} / \mathrm{min}$, an experimental regimen relevant for an anti-cancer drug delivery application. Valves significantly decreased reverse flow compared to a valve less system. We demonstrated that flow rate is not significantly affected by back pressures of up to $20 \mathrm{mmHg}$ (when delivering solutions of $1 \mathrm{cP}$ ) or delivering solutions with viscosities of up to $6 \mathrm{cP}$ (data shown in (Cobo, Tu et al. 2014)). As expected, micropump flow rate increased with increased environmental temperatures. Consistent micropump performance was also demonstrated in simulated physiological environmental conditions with less than $4 \%$ flow rate variation.

Drug delivery micropumps need to incorporate closed-loop feedback systems to enable pump performance monitoring, and increase effective therapies. Control over delivery profile can be achieved through the incorporation of physical sensors that provide information on pressure, flow rate, delivered volumes, forward flow delay and real-time state of the pump. A variety of sensing techniques are available for MEMS flow sensors, such as electrical, nuclear, thermal, mechanical and optical acoustic. We are currently developing an electrochemical impedance (EI) dose tracking system capable of tracking accidental release of reservoir content, blockage and refill detection or lack of delivery in real time (Sheybani, Cabrera-Munoz et al. 2012).

\section{Acknowledgements}

This work was funded in part by the NIH/NIGMS under award number R21GM104583, NSF PFI: AIR Technology Translation program under award number IIP-1343467, and the Viterbi School of Engineering Ph.D. Merit Fellowship (AC). The authors would like to thank the members of the USC Biomedical Microsystems Laboratory for their assistance with this project.

E. M. has a significant interest in Fluid Synchrony LLC (CA, USA).

\section{References}

Alzet. (2014). "ALZET® Osmotic Pumps - Implantable pumps for research." Retrieved 3/25/2014, from http://www.alzet.com. 
Broka, K. and P. Ekdunge (1997). "Oxygen and hydrogen permeation properties and water uptake of Nafion ${ }^{\circledR} 117$ membrane and recast film for PEM fuel cell." Journal of Applied Electrochemistry 27(2): 117-123.

Centers for Disease Control and Prevention. (2015). "World Cancer Day." Retrieved 7/14/15, from http://www.cdc.gov/cancer/dcpc/resources/features/worldcancerday/.

Cobo, A., R. Sheybani and E. Meng (2015). "MEMS: Enabled Drug Delivery Systems." Advanced healthcare materials 4(7): 969-982.

Cobo, A. M., H. M. Tu, R. Sheybani and E. Meng (2014). Characterization of a Wireless Implantable Infusion Micropump for Small Animal Research Under Simulated In Vivo Conditions. IEEE Biomedical Circuits and Systems, Lausanne, Switzerland.

DSM Somos Materials Group (2012). Somos ${ }^{\circledR W}$ aterShed XC 11122.

Gensler, H. and E. Meng (2012). "Rapid fabrication and characterization of MEMS Parylene C bellows for large deflection applications." Journal of Micromechanics and Microengineering 22(11): 115031.

Gensler, H., R. Sheybani, P.-Y. Li, R. L. Mann and E. Meng (2012). "An implantable MEMS micropump system for drug delivery in small animals." Biomedical Microdevices 14(3): 483-496.

Gensler, H., R. Sheybani and E. Meng (2013). A MEMS Micropump System with One-Way Valve for Chronic Drug Delivery. Microtechnologies in Medicine and Biology, Marina del Rey, CA.

Gensler, H., R. Sheybani, L. Po-Ying, R. Lo, S. Zhu, Y. Ken-Tye, I. Roy, P. N. Prasad, R. Masood, U. K. Sinha and E. Meng (2010). Implantable MEMS drug delivery device for cancer radiation reduction. 23rd IEEE International Conference on Micro Electro Mechanical Systems, MEMS 2010, Hong Kong, IEEE.

Gensler, H. M. (2013). A Wireless Implantable MEMS Micropump System for Site-specific Anti-cancer Drug Delivery, University of Southern California.

Ghodssi, R. and P. Lin (2011). MEMS materials and processes handbook, Springer Science \& Business Media.

Givrad, T. K. (2007). Induction power microbolus infusion pump used for functional neuroimaging applications in rodents, ProQuest.

Grigoriev, S., P. Millet, K. Dzhus, H. Middleton, T. Saetre and V. Fateev (2010). "Design and characterization of bi-functional electrocatalytic layers for application in PEM unitized regenerative fuel cells." international journal of hydrogen energy 35(10): 5070-5076.

Ioroi, T., K. Yasuda, Z. Siroma, N. Fujiwara and Y. Miyazaki (2002). "Thin film electrocatalyst layer for unitized regenerative polymer electrolyte fuel cells." Journal of Power sources 112(2): 583-587.

iPrecio. (2014). "iPRECIO $®$, Innovative Drug Infusion Technology for Laboratory Animals." Retrieved 3/25/2014, from http://www.iprecio.com.

John Urquhart, J. W. F., and Kay L. Willis (1984). "Rate-Controlled Delivery Systems in Drug and Hormone Research." Ann. Rev. Pharmacol. Toxicol 24: 199-236.

Kilinc, E. G., K. Kapucu, F. Maloberti and C. Dehollain (2014). Servo-controlled remote powering and low-power data communication of implantable bio-systems for freely moving animals. Biomedical Circuits and Systems Conference (BioCAS), 2014 IEEE, IEEE.

Langer, R. (1998). "Drug delivery and targeting." Nature 392(6679 Suppl): 5-10.

Lenaerts, B. and R. Puers (2009). Omnidirectional inductive powering for biomedical implants, Springer.

Lévi, F., C. Focan, A. Karaboué, V. de la Valette, D. Focan-Henrard, B. Baron, F. Kreutz and S. Giacchetti (2007). "Implications of circadian clocks for the rhythmic delivery of cancer therapeutics." Advanced Drug Delivery Reviews 59(9-10): 1015-1035.

Li, P.-Y., R. Sheybani, C. Gutierrez, J. T. Kuo and E. Meng (2010). "A parylene bellows electrochemical actuator." Microelectromechanical Systems, Journal of 19(1): 215-228.

Li, P.-Y., R. Sheybani, C. A. Gutierrez, J. T. W. Kuo and E. Meng (2010). "A Parylene bellows electrochemical actuator." Journal of Microelectromechanical Systems 19(Copyright 2010, The Institution of Engineering and Technology): 215-228.

Linder, C. C. and M. T. Davisson (2012). Chapter 1.2 - Historical Foundations. The Laboratory Mouse (Second Edition). H. J. Hedrich. Boston, Academic Press: 21-35.

Masood, R., I. Roy, S. Zu, C. Hochstim, K.-T. Yong, W.-C. Law, H. Ding, U. K. Sinha and P. N. Prasad (2012). "Gold nanorod-sphingosine kinase siRNA nanocomplexes: a novel therapeutic tool for potent radiosensitization of head and neck cancer." Integrative Biology 4(2): 132-141.

Med-E-Cell. (2014). "Welcome toMed-e-Cell: infusion pumps, fluid delivery devices, oxygen generation/control and fuel cell technology for licensing and development partnerships." Retrieved 3/25/2014, from http://medecell.com. 
Meng, E. and T. Hoang (2012). "MEMS-enabled implantable drug infusion pumps for laboratory animal research, preclinical, and clinical applications." Advanced Drug Delivery Reviews.

National Cancer Institute. (2015). "About Cancer." Retrieved 7/14/15, from http://www.cancer.gov/aboutcancer.

Neagu, C. R. (1998). A medical microactuator based on an electrochemical principle, Universiteit TwenteElektrotechniek.

Puers, R. (2008). Omnidirectional inductive powering for biomedical implants, Springer.

Reagan-Shaw, S., M. Nihal and N. Ahmad (2008). "Dose translation from animal to human studies revisited." The FASEB Journal 22(3): 659-661.

Sheybani, R., N. E. Cabrera-Munoz, T. Sanchez and E. Meng (2012). Design, fabrication, and characterization of an electrochemically-based dose tracking system for closed-loop drug delivery. Engineering in Medicine and Biology Society (EMBC), 2012 Annual International Conference of the IEEE.

Sheybani, R., H. Gensler and E. Meng (2012). "A MEMS electrochemical bellows actuator for fluid metering applications." Biomedical Microdevices: 1-12.

Sheybani, R. and E. Meng (2012). "High-Efficiency MEMS Electrochemical Actuators and Electrochemical Impedance Spectroscopy Characterization." Microelectromechanical Systems, Journal of 21(5): 1197-1208.

Sheybani, R. and E. Meng (2015). "Acceleration Techniques for Recombination of Gases in Electrolysis

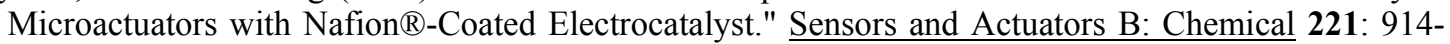
922.

Sheybani, R., Schober S. M., and Meng E. (2013). Drug Delivery Using Wireless MEMS. Handbook of MEMS for wireless and mobile applications. Uttamchandani, Woodhead Publishing: 489-517.

Smolensky, M. H. and N. A. Peppas (2007). "Chronobiology, drug delivery, and chronotherapeutics." Advanced Drug Delivery Reviews 59(9-10): 828-851.

Somos, D. (2008). "DSM Somos WaterShed XC 11122 Passes USP Class VI Testing: Now Suitable For Prototyping In Biomedical Or Skin Contact Applications." 2014, from http://www.meddeviceonline.com/doc/dsm-somos-watershed-xc-11122-passes-usp-0001.

The Johns Hopkins University. (2015). "Animal Care and Use Committee: The Mouse [and] The Rat." Retrieved 3/1/2015, from http://web.jhu.edu.libproxy.usc.edu/animalcare/procedures/.

Turner, P. V., T. Brabb, C. Pekow and M. A. Vasbinder (2011). "Administration of substances to laboratory animals: routes of administration and factors to consider." Journal of the American Association for Laboratory Animal Science: JAALAS 50(5): 600.

Turner, P. V., C. Pekow, M. A. Vasbinder and T. Brabb (2011). "Administration of substances to laboratory animals: equipment considerations, vehicle selection, and solute preparation." Journal of the American Association for Laboratory Animal Science: JAALAS 50(5): 614.

van Schuylenbergh, K. and R. Puers (2009). Inductive Powering: Basic Theory and Application to Biomedical Systems, Springer Science \& Business Media.

Youan, B.-B. C. (2004). "Chronopharmaceutics: gimmick or clinically relevant approach to drug delivery?" Journal of Controlled Release 98(3): 337-353.

\section{Figures}

Fig. 1 Schematic diagram of in vivo testing setup for small animal research with (a) commercial tethered infusion system, and (b) wireless implantable drug delivery pump

Fig. 2 Circuit schematic for the class E wireless inductive powering system. Link efficiency for concentric transmitting and receiving coils with zero angular misalignment was calculated to be approximately 0.82 .

Fig. 3 Schematic diagram of (a) drug delivery system, and (b) fully assembled wireless micropump

Fig. 4 Experimental setup for flow rate characterization of wireless micropumps 
Fig. 5 The effects of (a) axial separation distance between concentric transmitter and receiver coils, and (b) angular misalignment between concentric coils on pump performance (at $0.33 \mathrm{~mA}$ constant current)

Fig. 6 Representative data from one stationary micropump wirelessly operated. (a) Bolus delivery from a micropump operated once a day during week two (at $0.33 \mathrm{~mA}$ constant current) and (b) flow rates for the three weeks dosing

Fig. 7 Representative data from one wireless micropump flow rate performance when increasing the environmental temperature from room $\left(23{ }^{\circ} \mathrm{C}\right)$ to body temperature $\left(37^{\circ} \mathrm{C}\right)$ (at $0.33 \mathrm{~mA}$ constant current)

Fig. 8 Representative flow rate performance results for a micropump under simulated in vivo conditions for 30 days (at $0.33 \mathrm{~mA}$ constant current)

\section{Biographies}

Angelica M. Cobo received her B.S. degree in Biomedical Engineering from Florida International University in 2008. She was a research assistant in the Bioengineering Laboratory at the Diabetes Research Institute where she focused on the development of a bioactive scaffold material for use in cellular replacement therapies to treat Type 1 Diabetes Mellitus. In 2012, she received her M.S. in Biomedical Engineering from University of Southern California (USC). She is currently a $\mathrm{PhD}$ student in Biomedical Engineering at USC where she is working on the development of implantable MEMS drug delivery devices for management of chronic diseases in preclinical research.

Roya Sheybani received her B.S. (2008), M.S. (2009), PhD (2015) degrees in biomedical engineering from the University of Southern California (USC), Los Angeles, where she focused on developing closed-loop implantable wireless MEMS drug delivery devices for management of chronic diseases. She is currently a Hibbitt Engineering Postdoctoral fellow at Brown University, working towards developing "smart" biomedical devices that combine designer drug eluting biomaterials with biosensors to enable closed-loop therapies.

Heidi Tu received her B.S. in Biomedical Engineering and B.A. in German from The University of Texas at Austin. Heidi has a Ph.D. in Biomedical Engineering from the University of Southern California, where she developed an implantable drug delivery pump. She is currently a Product Design Engineer at a private healthcare technology company in the San Francisco Bay Area. Her professional goal is to develop medical technology to enable better treatment and care for patients.

Ellis Meng received her B.S. degree in engineering and applied science and her M.S. and Ph.D. degrees in electrical engineering from the California Institute of Technology (Caltech), Pasadena, in 1997, 1998, and 2003, respectively. She is a Professor of Biomedical Engineering and Electrical Engineering at the University of 
Southern California, Los Angeles where she has been since 2004. She directs the Biomedical Microsystems Laboratory which specializes in biomicroelectromechanical systems, implantable biomedical microdevices, neural interfaces, and microfluidics. She is a recipient of the NSF CAREER, the Wallace H. Coulter Foundation Early Career Translational Research, and the ASEE Curtis W. McGraw Research Awards. In 2009, she was recognized as one of the TR35 Technology Review Young Innovators under 35. E. M. has a significant financial interest in Fluid Synchrony LLC (CA, USA). 